\title{
A DELAY-DEPENDENT APPROACH TO ROBUST STABILITY FOR UNCERTAIN STOCHASTIC NEURAL NETWORKS WITH TIME-VARYING DELAY
}

Chien-Yu Lu

Department of Industrial Education and Technology, National Changhua University of Education, Changhua, Taiwan 500, R.O.C

Chin-Wen Liao

Department of Industrial Education and Technology, National Changhua University of Education, Changhua, Taiwan 500, R.O.C

Koan-Yuh Chang

Department of Electronic Engineering, Chienkuo Technology University, Changhua 500, Taiwan, R.O.C

Wen-Jer Chang

Department of Marine Engineering, National Taiwan Ocean University, Keelung 202, Taiwan, R.O.C., wjchang@mail.ntou.edu.tw

Follow this and additional works at: https://jmstt.ntou.edu.tw/journal

Part of the Electrical and Computer Engineering Commons

\section{Recommended Citation}

Lu, Chien-Yu; Liao, Chin-Wen; Chang, Koan-Yuh; and Chang, Wen-Jer (2010) "A DELAY-DEPENDENT APPROACH TO ROBUST STABILITY FOR UNCERTAIN STOCHASTIC NEURAL NETWORKS WITH TIME-VARYING DELAY," Journal of Marine Science and Technology. Vol. 18: Iss. 1, Article 9.

DOI: $10.51400 / 2709-6998.1867$

Available at: https://jmstt.ntou.edu.tw/journal/vol18/iss1/9

This Research Article is brought to you for free and open access by Journal of Marine Science and Technology. It has been accepted for inclusion in Journal of Marine Science and Technology by an authorized editor of Journal of Marine Science and Technology. 


\title{
A DELAY-DEPENDENT APPROACH TO ROBUST STABILITY FOR UNCERTAIN STOCHASTIC NEURAL NETWORKS WITH TIME-VARYING DELAY
}

\author{
Chien-Yu Lu*, Chin-Wen Liao*, Koan-Yuh Chang**, and Wen-Jer Chang***
}

Key words: time-varying delay, linear matrix inequality, neural networks, uncertainty, robust stability.

\begin{abstract}
This paper investigates the global delay-dependent robust stability in the mean square for uncertain stochastic neural networks with time-varying delay. The activation functions are assumed to be globally Lipschitz continuous. Based on a linear matrix inequality approach, globally delay-dependent robust stability criterion is derived by introducing some relaxation matrices which, when chosen properly, lead to a less conservative result. Two numerical examples are given to illustrate the effectiveness of the method.
\end{abstract}

\section{INTRODUCTION}

Recently, it has been well-known that time delays are frequently encountered in various electronic implementation of neural networks with time delay, such as, Hopfield neural networks, cellular neural networks and bi-directional associative memory networks, and its existence is a source of oscillation and instability of neural networks. Therefore, the research of the dynamical characteristics (include stable, unstable, oscillatory and chaotic behavior) of neural networks with time delays is an important topic in the neural networks theory. In particular, globally stability is one of the most desirable dynamic properties of neural networks, there have been growing research interest on the stability analysis problem for delayed neural networks. Recently, considerable efforts have been extensively applied to the analysis of the stability in

Paper submitted 08/29/08; revised 02/12/09; accepted 02/16/09. Author for correspondence: Wen-Jer Chang (e-mail: wjchang@mail.ntou.edu.tw).

*Department of Industrial Education and Technology, National Changhua University of Education, Changhua, Taiwan 500, R.O.C.

**Department of Electronic Engineering, Chienkuo Technology University, Changhua 500, Taiwan, R.O.C.

***Department of Marine Engineering, National Taiwan Ocean University, Keelung 202, Taiwan, R.O.C. signal and image processing, artificial intelligence, industrial automation, and other fields $[2,6,7,12,19]$. It is noted that, so far, most works on delayed neural networks have dealt with the stability analysis problem for neural networks with time delays.

Much of the current interest in neural networks stems not only from their richness as a theoretical model of collective dynamics but also from the promise they have shown as a practical tool for performing parallel computation. In carrying out the computation, there are various stochastic perturbations to the networks and it is important to understand how these perturbations affect the networks. Consequently, it is very urgent to know whether the networks are stable or not under the perturbations. According, the stability criteria for stochastic neural networks become an attractive research problem of prime importance. Lately, some initial results have just appeared, for example, the stability problem for the stochastic neural networks without delays has been studied in [13], and stochastic delayed neural networks has been discussed in [3, $11,16,17,22]$. The stability analysis of stochastic delayed cellular neural networks in terms of linear matrix inequality [5] approach was proposed in [22]. By using the method of variation parameter, inequality technique and stochastic analysis, the sufficient conditions to guarantee the mean square exponential stability of an equilibrium solution are given in [17]. It should be pointed out that the aforementioned results in $[3,8$, $16,17,21]$ were obtained as delay-independent conditions, that is, delay-independent conditions do not include any information relating to the magnitude of the delays. However, it is known that delay-dependent conditions are generally less conservative than delay-independent conditions; particularly when the magnitude of the delay is small. Although delayindependent results on the robust stability problem for stochastic neural networks with discrete delay and mixed delays were presented in [21] and [18], respectively, no delaydependent robust stability results on stochastic neural networks with time-varying delay are available in the literature, which motivates the present study.

In this paper, we are concerned with the global delaydependent robust stability conditions in the mean square for 
stochastic uncertain neural networks with time-varying delay. Some criteria on globally delay-dependent robust stability conditions are presented. Based on Lyapunov-Krasovskii functional combining with linear matrix inequality (LMI) techniques, globally delay-dependent robust stability conditions for stochastic uncertain neural networks with timevarying delay, which are given in terms of quadratic forms of state and LMI, are derived. We will also introduce some relaxation matrices which, when chosen properly, produce a less conservative result $[10,14,20]$. A numerical example will be finally given to determine the effectiveness of the method.

Notation: Throughout this paper, the notation $X \geq Y$ (respectively $X>Y$ ) for symmetric matrices $X$ and $Y$ means that the matrix $X-Y$ is positive semidefinite (respectively, positive definite), $Z^{T}$ represents the transpose of the matrix $Z$. The vector norm $\|\cdot\|$ refers to the Euclidean vector norm, that is $\|W\|=\lambda_{M}^{1 / 2}\left(W^{T} W\right)$, where $\lambda_{M}(W)$ (respectively $\lambda_{m}(W)$ ) stands for the operation of taking the maximum (respectively, minimum) eigenvalue of $W$.

\section{SYSTEM DESCRIPTION AND PROBLEM FORMULATION}

The delayed neural network model is defined by the following uncertain state equations with time-varying delay

$$
\begin{aligned}
\dot{u}(t)= & -(A+\Delta A(t)) u(t)+(W+\Delta W(t)) g(u(t)) \\
& +\left(W_{1}+\Delta W_{1}(t)\right) g(u(t-\tau(t))),
\end{aligned}
$$

or equivalently

$$
\begin{aligned}
\dot{u}_{i}(t)= & -\left(a_{i}+\Delta a_{i}(t)\right) u_{i}(t)+\sum_{j=1}^{n}\left(w_{i j}+\Delta w_{i j}(t)\right) g_{j}\left(u_{j}(t)\right) \\
& +\sum_{j=1}^{n}\left(w_{1 i j}+\Delta w_{1 i j}(t)\right) g_{j}\left(u_{j}(t-\tau(t))\right), i=1,2, \ldots, n,
\end{aligned}
$$

where $u(t)=\left[u_{1}(t), u_{2}(t), \ldots, u_{n}(t)\right]^{T}$ is the state vector of the neural network, $A=\operatorname{diag}\left(a_{1}, a_{2}, \ldots, a_{n}\right)$ is a diagonal matrix with positive entries, $a_{i}>0, i=1, \ldots, n, W=\left[w_{i j}\right]_{n \times n}$, and $W_{1}=$ $\left[w_{1 i j}\right]_{n \times n}$ represent the connection weight matrix of the neurons and the delayed connection weight matrix of the neurons, respectively, $g(u(t))=\left[g_{1}\left(u_{1}(t), g_{2}\left(u_{2}(t), \ldots, g_{n}\left(u_{n}(t)\right)\right]^{T}\right.\right.$ denotes the neuron activation function with $\mathrm{g}(0)=0, \tau(t)$ is the transmission delay satisfying $0 \leq \tau(t) \leq \tau$ and $\dot{\tau}(t) \leq d$.

In practical implementation of neural networks, the values of the constant $a_{i}$ and weight coefficients $w_{i j}$ and $w_{1 i j}$ depend on certain resistance and capacitance values which are subject to uncertainties. This may trigger some deviations in the values of $a_{i}, w_{i j}$ and $w_{1 i j}$. So, $\Delta A(t), \Delta W(t)$ and $\Delta W_{1}(t)$ are unknown matrices representing time-varying parameter uncertainties, and are assumed to be of the form

$$
\left[\Delta A(t) \quad \Delta W(t) \quad \Delta W_{1}(t)\right]=H F(t)\left[\begin{array}{lll}
E_{1} & E_{2} & E_{3}
\end{array}\right]
$$

where $H, E_{1}, E_{2}$ and $E_{3}$ are known real constant matrices and $F(t)$ is an unknown real-valued time-varying matrix satisfying

$$
F^{T}(t) F(t) \leq I, \forall t
$$

It is assumed that all the elements of $F(t)$ are Lebesgue measurable. $\Delta A(t), \Delta W(t)$ and $\Delta W_{1}(t)$ are said to be admissible if both (3) and (4) hold.

Suppose there exists a stochastic perturbation to the neural network and the stochastically perturbed network is described by a stochastic system with time-varying delay

$$
\begin{aligned}
d x(t)= & {[-(A+\Delta A(t)) x(t)+(W+\Delta W(t)) g(x(t))} \\
& \left.+\left(W_{1}+\Delta W_{1}(t)\right) g(x(t-\tau(t)))\right] d t \\
& +\sigma(x(t), x(t-\tau(t))) d B(t),
\end{aligned}
$$

with initial data

$$
x(t)=\varphi(t), \forall t \in[-\tau, 0] .
$$

Here $B(t)$ is an n-dimensional Brownian motion defined on the given complete probability space. We assume that $\sigma(\cdot)$ : $R^{n} \times R^{n} \rightarrow R^{n \times m}$ is locally Lipschitz continuous and satisfies linear growth condition.

$$
\left\|\sigma\left(x_{1}, x_{2}\right)-\sigma\left(y_{1}, y_{2}\right)\right\| \leq\left\|G_{1}\left(x_{1}-y_{1}\right)\right\|+\left\|G_{2}\left(x_{2}-y_{2}\right)\right\|,
$$

for all $x_{1}, x_{2}, y_{1}$, and $y_{2} \in R^{n}$, where $G_{1}$ and $G_{2}$ are known real constant matrices. Equation (5) has a unique global solution on $t \geq 0$ and we show the solution by $x(t ; \varphi)$. Also, we assume that $\sigma(0,0)=0$ for the stability purpose and hence (5) permits a trivial solution $x(t ; 0)=0$ [8].

\section{Assumption 1 (Lipschitz condition):}

The activation function $g(x)$ is nondecreasing, bounded that is

$$
0 \leq \frac{g_{j}\left(\xi_{1}\right)-g_{j}\left(\xi_{2}\right)}{\xi_{1}-\xi_{2}} \leq \alpha_{j}, \quad \xi_{1} \neq \xi_{2}, \quad j=1, \cdots, n
$$

The following definition is necessary in the theory of stochastic differential equations [15].

Definition 1: If there exist $\beta>0$ and $\gamma>0$ such that

$$
E\|x(t)\|^{2} \leq \gamma \sup _{-\tau \leq s \leq 0} E\|\varphi(s)\|^{2} e^{-\beta t}, t>0
$$

Then, the system (5) is said to be exponentially mean square stable 
The main results of this paper hinge on the following fact.

Fact 1 [9]: The trivial solution of a stochastic delayed differential equation

$$
\begin{gathered}
d x(t)=F(x(t), x(t-\tau(t)), t) d t+G(x(t), x(t-\tau(t)), t) d w(t) \\
x(t)=\varphi(t), \quad \forall t \in[-\tau, 0]
\end{gathered}
$$

on $t \in\left[t_{0}, T\right]$ with initial data

$f(\varphi, t)=F(\varphi(0), \varphi(-\tau), t)$ and $g(\varphi, t)=G(\varphi(0), \varphi(-\tau), t)$,

where $F: R^{n} \times R^{n} \times\left[t_{0}, T\right] \rightarrow R^{n}$ and $G: R^{n} \times R^{n} \times\left[t_{0}, T\right] \rightarrow$ $R^{n \times m}$ for $(\varphi, t) \in \mathrm{C}\left([-\tau, 0] ; R^{n}\right) \times\left[t_{0}, T\right]$, is globally asymptotically stable in probability if there exists a function $V(t, x) \in$ $C_{2}\left(U_{+} \times R^{n}\right)$ which is positive definite in the Lyapunov sense, and satisfies the generator $L V$

$$
L V=\frac{\partial V}{\partial t}+(\operatorname{grad} V) F+\frac{1}{2}\left(\operatorname{tr} G G^{T}\right) H e s s V<0,
$$

for $x \neq 0$ and $V(t, x) \rightarrow+\infty$ as $|x| \rightarrow \infty$. The matrix Hess $V$ is the Hessian matrix of the second-order partial derivatives. This fact is analogous to the well-known theorem of Lyapunov for deterministic systems.

\section{MAIN RESULTS}

In this section, the exponentially mean-square stability for uncertain stochastic system (5) with time-varying delays is explored. An LMI approach is developed to solve the robust stochastic stability if the system associated to (5) is meansquare asymptotically stable for all admissible uncertainties $\Delta A(t), \Delta W(t)$ and $\Delta W_{1}(t)$. The analysis first gives some results which are essential to introduce the following Lemma 1 for the development of our main theorem.

Lemma 1 [4]: For any vectors or matrices $z$ and $y$ with appropriate dimensions and any positive constant $\varepsilon$, the following inequality is satisfied:

$$
-2 z^{T} y \leq \varepsilon z^{T} z+\varepsilon^{-1} y^{T} y .
$$

Define a new state variable

$$
\begin{aligned}
z(t)= & -(A+\Delta A(t)) x(t)+(W+\Delta W(t)) g(x(t)) \\
& +\left(W_{1}+\Delta W_{1}(t)\right) g(x(t-\tau(t))) .
\end{aligned}
$$

Equation (5) can be rewritten as

$$
d x(t)=z(t) d t+\sigma(x(t), x(t-\tau(t))) d B(t)
$$

For any matrices $N_{i}$ and $S_{i}(i=1,2,3,4,5)$ of appropriate dimensions, it can be shown that

$$
\begin{gathered}
{\left[x^{T}(t) N_{1}+x^{T}(t-\tau(t)) N_{2}+g^{T}(x(t)) N_{3}+g^{T}\left(x(t-\tau(t)) N_{4}\right.\right.} \\
\left.+z^{T}(t) N_{5}\right] \times\left[x(t)-x(t-\tau(t))-\int_{t-\tau(t)}^{t} d x(s)\right]=0
\end{gathered}
$$

$$
\begin{aligned}
& {\left[x^{T}(t) S_{1}+x^{T}(t-\tau(t)) S_{2}+g^{T}(x(t)) S_{3}+g^{T}\left(x(t-\tau(t)) S_{4}\right.\right.} \\
& \left.\quad+z^{T}(t) S_{5}\right] \times\{[-(A+\Delta A(t)) x(t)+(W+\Delta W(t)) g(x(t)) \\
& \left.\left.\quad+\left(W_{1}+\Delta W_{1}(t)\right) g(x(t-\tau(t)))\right]-z(t)\right\} .
\end{aligned}
$$

Theorem 1: For any given $\tau>0$ and $0 \leq d<1$ satisfying $0 \leq \tau(t) \leq \tau$ and $\dot{\tau}(t) \leq d$, if there exist matrices $P>0, R>0$, $Q_{1}>0, Q_{2}>0, S_{5}<0, X_{11} \geq 0, X_{22} \geq 0, X_{33} \geq 0, X_{44} \geq 0, X_{55} \geq 0$, $X_{12} \geq 0, X_{13} \geq 0, X_{14} \geq 0, X_{15} \geq 0, X_{23} \geq 0, X_{24} \geq 0, X_{25} \geq 0, X_{34} \geq$ $0, X_{35} \geq 0, X_{45} \geq 0$ and diagonal matrix $T>0$ and any matrices $N_{i}(i=1,2,3,4,5), S_{1}, S_{2}, S_{3}$ and $S_{4}$ of appropriate dimensions and positive scalars $\varepsilon_{1}, \varepsilon_{2}$ and $\rho$ such that

$$
\begin{gathered}
{\left[\begin{array}{lrc}
(1,1) & N & S H \\
N^{T} & -\varepsilon_{1} & 0 \\
H^{T} S^{T} & 0 & -\varepsilon_{2}
\end{array}\right]<0,} \\
{\left[\begin{array}{ll}
X & N \\
N^{T} & R
\end{array}\right] \geq 0,}
\end{gathered}
$$

and

$$
P \leq \rho I
$$

Then the system described by (5) is exponentially stable in the mean square, where

$$
(1,1)=\Pi+\tau X
$$

$$
\Pi=\left[\begin{array}{ccccc}
\Pi_{11} & \Pi_{12} & \Pi_{13} & \Pi_{14} & \Pi_{15} \\
\Pi_{12}^{T} & \Pi_{22} & \Pi_{23} & \Pi_{24} & \Pi_{25} \\
\Pi_{13}^{T} & \Pi_{23}^{T} & \Pi_{33} & \Pi_{34} & \Pi_{35} \\
\Pi_{14}^{T} & \Pi_{24}^{T} & \Pi_{34}^{T} & \Pi_{44} & \Pi_{45} \\
\Pi_{15}^{T} & \Pi_{25}^{T} & \Pi_{35}^{T} & \Pi_{45}^{T} & \Pi_{55}
\end{array}\right],
$$




$$
\begin{array}{ll}
\Pi_{11}=Q_{1}+\left(\tau \varepsilon_{1}+\delta\right) G_{1}^{T} G_{1}+N_{1}+N_{1}^{T}+\varepsilon_{2} E_{1}^{T} E_{1}+S_{1} A+A^{T} S_{1}^{T}, & V_{4}(x(t), t)=\int_{t-\tau}^{t} \int_{s}^{t} \varepsilon_{1}\left[\left\|G_{1} x(v)\right\|^{2}+\left\|G_{2} x(v-\tau(v))\right\|^{2}\right] d v d s, \\
\Pi_{12}=N_{2}^{T}-N_{1}+A^{T} S_{2}^{T}, & V_{5}(x(t), t)=\int_{t-\tau}^{t} \int_{s}^{t} z^{T}(v) R z(v) d v d s .
\end{array}
$$

$\Pi_{14}=N_{4}^{T}-S_{1} W_{1}+A^{T} S_{4}^{T}+\varepsilon_{2} E_{1}^{T} E_{3}$,

$\Pi_{15}=N_{5}^{T}+P+S_{1}+A^{T} S_{5}^{T}$,

$\Pi_{22}=-(1-d) Q_{1}+\left(\tau \varepsilon_{1}+\delta\right) G_{2}^{T} G_{2}-N_{2}-N_{2}^{T}$,

$\Pi_{23}=-S_{2} W-N_{3}^{T}, \Pi_{24}=-S_{2} W_{1}-N_{4}^{T}, \quad \Pi_{25}=S_{2}-N_{5}^{T}$,

$\Pi_{33}=Q_{2}-T-T^{T}-S_{3} W-W^{T} S_{3}^{T}+\varepsilon_{2} E_{2}^{T} E_{2}$,

$\Pi_{34}=N_{4}^{T}-S_{3} W_{1}-W^{T} S_{4}^{T}+\varepsilon_{2} E_{2}^{T} E_{3}, \quad \Pi_{35}=S_{3}-W^{T} S_{5}^{T}$,

$\Pi_{44}=-(1-d) Q_{2}+\varepsilon_{2} E_{3}^{T} E_{3}-S_{4} W_{1}-W_{1}^{T} S_{4}^{T}$,

$\Pi_{45}=S_{4}-W_{1}^{T} S_{5}^{T}, \quad \Pi_{55}=S_{5}+S_{5}^{T}+\tau R$,

$$
X=\left[\begin{array}{ccccc}
X_{11} & X_{12} & X_{13} & X_{14} & X_{15} \\
X_{12}^{T} & X_{22} & X_{23} & X_{24} & X_{25} \\
X_{13}^{T} & X_{23}^{T} & X_{33} & X_{34} & X_{35} \\
X_{14}^{T} & X_{24}^{T} & X_{34}^{T} & X_{44} & X_{45} \\
X_{15}^{T} & X_{25}^{T} & X_{35}^{T} & X_{45}^{T} & X_{55}
\end{array}\right],
$$

$$
N=\left[\begin{array}{lllll}
N_{1}^{T} & N_{2}^{T} & N_{3}^{T} & N_{4}^{T} & N_{5}^{T}
\end{array}\right]^{T}, S=\left[\begin{array}{lllll}
S_{1}^{T} & S_{2}^{T} & S_{3}^{T} & S_{4}^{T} & S_{5}^{T}
\end{array}\right]^{T}
$$

Proof: Consider the following Lyapunov-Krasovskii functional for the system (5)

$$
\begin{aligned}
V(x(t), t)= & V_{1}(x(t), t)+V_{2}(x(t), t)+V_{3}(x(t), t)+V_{4}(x(t), t) \\
& +V_{5}(x(t), t)
\end{aligned}
$$

where

$$
\begin{gathered}
V_{1}(x(t), t)=x^{T}(t) P x(t), \\
V_{2}(x(t), t)=\int_{t-\tau(t)}^{t} x^{T}(s) Q_{1} x(s) d s, \\
V_{3}(x(t), t)=\int_{t-\tau(t)}^{t} g^{T}(x(s)) Q_{2} g(x(s)) d s,
\end{gathered}
$$

Along trajectories of (5) and making use of the It ô differential rule [9], then the generator $L V(x(t), t)$ for the evolution of $V(x(t), t)$ is given by

$$
\begin{aligned}
& L V(x(t), t) \\
& \leq 2 x^{T}(t) P z(t) \\
& +\operatorname{Trace}\left[\sigma^{T}(x(t), x(t-\tau(t))) P \sigma(x(t), x(t-\tau(t)))\right]+x^{T}(t) Q_{1} x(t) \\
& -(1-d) x^{T}(t-\tau(t)) Q_{1} x(t-\tau(t))+g^{T}(x(t)) Q_{2} g(x(t)) \\
& -(1-d) g^{T}(x(t-\tau(t))) Q_{2} g(x(t-\tau(t)))+\tau \varepsilon_{1}\left[\left\|G_{1} x(t)\right\|^{2}+\left\|G_{2} x(t-\tau(t))\right\|^{2}\right] \\
& -\int_{t-\tau}^{t} \varepsilon_{1}\left[\left\|G_{1} x(s)\right\|^{2}+\left\|G_{2} x(s-\tau(s))\right\|^{2}\right] d s+\tau z^{T}(t) R z(t)-\int_{t-\tau}^{t} z^{T}(s) R z(t) d s \\
& +2\left[x^{T}(t) N_{1}+x^{T}(t-\tau(t)) N_{2}+g^{T}(x(t)) N_{3}+g^{T}\left(x(t-\tau(t)) N_{4}+z^{T}(t) N_{5}\right]\right. \\
& \times\left[x(t)-x(t-\tau(t))-\int_{t-\tau(t)}^{t} d x(s)\right] \\
& -2\left[x^{T}(t) S_{1}+x^{T}(t-\tau(t)) S_{2}+g^{T}(x(t)) S_{3}+g^{T}\left(x(t-\tau(t)) S_{4}+z^{T}(t) S_{5}\right]\right. \\
& \times\{[-(A+\Delta A(t)) x(t)+(W+\Delta W(t)) g(x(t)) \\
& \left.\left.+\left(W_{1}+\Delta W_{1}(t)\right) g(x(t-\tau(t)))\right]-z(t)\right\}+\tau q^{T}(t) X q(t) \\
& -\int_{t-\tau(t)}^{t} q^{T}(t) X q(t) d \alpha-2 g^{T}(x(t)) \operatorname{Tg}(x(t))+2 g^{T}(x(t)) \operatorname{Tg}(x(t)),
\end{aligned}
$$

where

$q(t)=\left[\begin{array}{lllll}x^{T}(t) & x^{T}(t-\tau(t)) & g^{T}(x(t)) & g^{T}(x(t-\tau(t))) & z^{T}(t)\end{array}\right]^{T}$,

$X \geq 0$ and diagonal matrix $T>0$.

It follows from Lemma 1 that for any $\varepsilon_{1}>0$ and $\varepsilon_{2}>0$, 


$$
\begin{aligned}
& -2\left[x^{T}(t) N_{1}+x^{T}(t-\tau(t)) N_{2}+g^{T}(x(t)) N_{3}\right. \\
& +g^{T}\left(x(t-\tau(t)) N_{4}+g^{T}(t) N_{5}\right] \int_{t-\tau(t)}^{t} d x(s) \\
& \leq-2 q^{T}(t) N \int_{t-\tau(t)}^{t} z(s) d s+q^{T}(t) \varepsilon_{1}^{-1} N N^{T} q(t) \\
& +\varepsilon_{1} \| \int_{t-\tau(t)}^{t} \sigma\left(x(s), x(s-\tau(s)) d B(s) \|^{2},\right.
\end{aligned}
$$

$-2 q^{T}(t) S H F(t) E q(t) \leq q^{T} \varepsilon_{2}^{-1} S H H^{T} S^{T} q(t)+\varepsilon_{2} q^{T}(t) \tilde{E}^{T} \tilde{E} q(t)$,

with $\tilde{E}=\left[\begin{array}{lllll}E_{1} & 0 & E_{2} & E_{3} & 0\end{array}\right]$.

Next, it follows from the condition (7) and (20) that

$$
\begin{aligned}
& \text { Trace }\left[\sigma^{T}(x(t), x(t-\tau(t))) P \sigma(x(t), x(t-\tau(t)))\right] \\
& \leq x^{T}(t) \rho G_{1}^{T} G_{1} x(t)+x^{T}(t-\tau(t)) \rho G_{2}^{T} G_{2} x(t-\tau(t)),
\end{aligned}
$$

where $\rho=\lambda_{\max }(P)$.

Using Assumption 1 yields

$$
2 g^{T}(x(t)) H g(x(t)) \leq 2 g^{T}(x(t)) H \Gamma x(t),
$$

where $\Gamma=\operatorname{diag}\left(\alpha_{1}, \alpha_{2}, \cdots, \alpha_{n}\right)$.

Moreover,

$E \| \int_{t-\tau(t)}^{t} \sigma\left(x(s), x(s-\tau(s)) d B(s) \|^{2} \leq \int_{t-\tau(t)}^{t} E\left[\left\|G_{1} x(s)\right\|^{2}+\left\|G_{2} x(s-\tau(s))\right\|^{2}\right] d s\right.$

Combining now (27)-(32) yields

$E\{L V(x(t), t)\} \leq E\left\{q^{T}(t) \Pi_{1} q(t)\right\}-\int_{t-\tau(t)}^{t} E\left\{q^{T}(t, \mathrm{~s}) \Pi_{2} q(t, s)\right\} d s$,

with $q^{T}(t, s)=\left[\begin{array}{ll}q^{T}(t) & z^{T}(s)\end{array}\right]$.

Next, from (18) and (19), we can show $L V<0$.

Define a new function as

$$
Y(x(t), t)=e^{\beta t} V(x(t), t),
$$

its infinitesimal operator $L$ is given by

$$
L Y(x(t), t)=\beta e^{\beta t} V(x(t), t)+e^{\beta t} L V(x(t), t) .
$$

By integrating this relation both sides between 0 to $t$ and then taking expectation yield

$$
\begin{aligned}
E & \{Y(x(t), t)\}-E\{Y(x(0), 0)\} \\
& =\int_{0}^{t} \beta e^{\beta \alpha} E\{V(x(s), s)\} d s+\int_{0}^{t} e^{\beta \alpha} E\{L V(x(s), s)\} d s
\end{aligned}
$$

By using the similar analysis method in [15], it can be seen from (21), (34) and (36) that, if $\beta>0$ is chosen small enough, a constant $\gamma>0$ can be found such that

$$
E\{V(x(t), t)\} \leq \gamma \sup _{-\tau \leq s \leq 0} E\left\{\|\varphi(s)\|^{2}\right\} e^{-\beta t}
$$

It follows from (21) that

$$
V(x(t), t) \geq \delta x^{T}(t) x(t)
$$

where $\delta=\min \left\{\lambda_{\min }(P)\right\}$, it can further imply from (37) that

$$
E\left\{x^{T}(t) x(t)\right\} \leq \bar{\gamma} \sup _{-\tau \leq s \leq 0} E\left\{\|\varphi(s)\|^{2}\right\} e^{-\beta t},
$$

where $\bar{\gamma}=\delta^{-1} \gamma$. From this the result follows.

Remark 1: This paper is concerned with the global delaydependent mean square exponential robust stability for stochastic uncertain neural networks with time-varying delay. The delayed neural networks via LMI approach in $[1,14]$ can be regarded as the speciality of the stochastic delayed neural networks. Therefore, the global asymptotic stability presented in $[1,14]$ is the specialization of Theorem 1 obviously.

Let us now work out two numerical examples to show the usefulness of the proposed results.

\section{NUMERICAL EXAMPLES}

Example 1: Consider a stochastic uncertain delayed neural network

$$
\begin{aligned}
d x(t)= & {[-(A+\Delta A(t)) x(t)+(W+\Delta W(t)) g(x(t))} \\
& \left.+\left(W_{1}+\Delta W_{1}(t)\right) g(x(t-\tau(t)))\right] d t \\
& +\sigma(x(t), x(t-\tau(t))) d B(t),
\end{aligned}
$$


where

$A=\left[\begin{array}{ll}2 & 0 \\ 0 & 0.9\end{array}\right], W=\left[\begin{array}{lr}-1 & 0 \\ -0.5 & -1\end{array}\right], W_{1}=\left[\begin{array}{ll}0.9 & -1.2 \\ 0.05 & -0.9\end{array}\right]$,

$H=\left[\begin{array}{cc}0.1 & 0 \\ 0 & 0.3\end{array}\right], E_{1}=\left[\begin{array}{cc}0.02 & 0.02 \\ -0.1 & 0.2\end{array}\right], E_{2}=\left[\begin{array}{cc}-0.07 & 0.3 \\ -0.01 & 0.2\end{array}\right]$

$E_{3}=\left[\begin{array}{cc}0.02 & -0.02 \\ 0.1 & -0.1\end{array}\right], G_{1}=G_{2}=\left[\begin{array}{cc}\sqrt{0.1} & 0 \\ 0 & \sqrt{0.1}\end{array}\right]$

The activation function is assumed to satisfy $\alpha_{1}=0.5$ and $\alpha_{2}=0.3$ in Assumption 1 .

Then, it can be shown that the global delay-dependent robust conditions in $[3,5,22]$ cannot be satisfied for any $\tau>0$. Accordingly, they cannot provide any results on the maximum allowed delay $\tau$. However, utilizing Theorem 1 in this paper, the maximum allowable value of $\tau$ for different $d$ can be got as follows.

\begin{tabular}{|c|c|}
\hline$d$ & Maximum allowable value of $\tau$ \\
\hline 0 & 5.38 \\
\hline 0.5 & 4.5 \\
\hline 0.9 & 3.26 \\
\hline
\end{tabular}

Therefore, the stochastic uncertain neural networks with time-varying delay in Theorem 1 of this paper are less conservative than those results in $[3,5,22]$.

Example 2: Consider a stochastic uncertain delayed neural network in (5) with parameters

$$
\begin{aligned}
& A=\left[\begin{array}{ccc}
1.1 & 0 & 0 \\
0 & 2.1 & 0 \\
0 & 0 & 2.9
\end{array}\right], W=\left[\begin{array}{ccc}
-3.4 & 0.1 & 0.4 \\
-3.9 & 4.8 & 1.9 \\
-0.9 & -0.6 & -1.1
\end{array}\right], \\
& W_{1}=\left[\begin{array}{rrr}
-3.4 & 0 & 0 \\
0 & -4.8 & 0 \\
0 & 0 & 3.5
\end{array}\right], H=\left[\begin{array}{ccc}
0.1 & 0 & 0 \\
0 & 0.2 & 0 \\
0 & 0 & 0.3
\end{array}\right], \\
& E_{1}=\left[\begin{array}{rrr}
0.2 & 0.2 & 0.1 \\
-0.1 & 0.2 & 0.3 \\
0.3 & 0.2 & 0.1
\end{array}\right], E_{2}=\left[\begin{array}{ccc}
-0.1 & 0.3 & 0.2 \\
-0.1 & 0.2 & 0.3 \\
0.2 & 0.1 & 0.3
\end{array}\right], \\
& E_{3}=\left[\begin{array}{ccc}
0.2 & -0.2 & 0.3 \\
0.1 & -0.1 & 0.2 \\
0.2 & 0.1 & 0.3
\end{array}\right], G_{1}=G_{2}=\left[\begin{array}{ccc}
\sqrt{0.1} & 0 & 0 \\
0 & \sqrt{0.1} & 0 \\
0 & 0 & \sqrt{0.1}
\end{array}\right] .
\end{aligned}
$$

The activation function is assumed to satisfy Assumption 1 with $\alpha_{1}=0.3, \alpha_{2}=0.4$ and $\alpha_{3}=0.5$. Furthermore, the time delay is assumed to satisfy $0<\dot{\tau}(t) \leq 0.5$. For the stochastic uncertain neural networks with time-varying delay, it is found that the conditions in $[3,11,17,21]$ and [21] are not satisfied for any $\tau>0$. Accordingly, they fail to conclude whether this stochastic uncertain neural network with time-varying delay is globally delay-dependent robust stability. However, by using Matlab LMI Control Toolbox, it can be verified that this stochastic uncertain neural network with time-varying delay is globally delay-dependent robust stability for all $0<\tau(t) \leq 6.5$. In this case, the solution can be got as

$$
\begin{aligned}
& P=\left[\begin{array}{ccc}
1.9301 & 0 & 0 \\
0 & 1.9301 & 0 \\
0 & 0 & 1.9302
\end{array}\right] \\
& Q_{1}=\left[\begin{array}{rrr}
4.5589 & -0.0029 & 0.0018 \\
-0.0029 & 4.5489 & -0.0003 \\
0.0018 & -0.0003 & 4.5238
\end{array}\right] \\
& Q_{2}=\left[\begin{array}{rrr}
1.7730 & -0.0314 & -0.0501 \\
-0.0314 & 1.3677 & 0.0881 \\
-0.0501 & 0.0881 & 1.6200
\end{array}\right] \\
& R=\left[\begin{array}{rrr}
0.3715 & -0.0262 & -0.2341 \\
-0.0262 & 0.0286 & 0.0624 \\
-0.2341 & 0.0624 & 0.5811
\end{array}\right] \\
& C=\left[\begin{array}{ccc}
4.6884 & 0 & 0 \\
0 & 0.2568 & 0 \\
0 & 0 & 3.2044
\end{array}\right], T=\left[\begin{array}{ccc}
2.3592 & 0 & 0 \\
0 & 5.8334 & 0 \\
0 & 0 & 3.1481
\end{array}\right], \\
& \varepsilon_{1}=1.7392, \varepsilon_{2}=2.2728 \text {. }
\end{aligned}
$$

Therefore, by Theorem 1 in this paper, this stochastic uncertain neural network with time-varying delay is globally robust delay-dependent stability, which implies that for this example Theorem 1 in this paper can be less conservative that the existing results in the literature.

\section{CONCLUSIONS}

In this paper, the problem of globally delay-dependent robust stability for a class of stochastic neural networks with time-varying delay has been considered. A sufficient condition for the solvability of this problem, which depends on the size of the time delay, has been presented by means of the Lyapunov-Krasovskii functional and the LMI approach. Two 
numerical examples have shown the effectiveness of the proposed approach.

\section{REFERENCES}

1. Arik, S., "An analysis of exponential stability of delayed neural networks with time varying delays," Neural Networks, Vol. 17, pp. 1027-1031 (2002).

2. Baldi, P. and Atiya, A. F., "How delays affect neural dynamics and learning," IEEE Transactions on Neural Networks, Vol. 5, pp. 612-621 (1994).

3. Blythe, S., Mao, X., and Liao, X. X., "Stability of stochastic delay neural networks," Journal of The Franklin Institute, Vol. 338, pp. 481-495 (2001).

4. Boukas, E. K. and Liu, Z. K., Deterministic and Stochastic Time Delay Systems, Birkhauser, Boston, 2002.

5. Boyd, S., Ghaoui, L. Ei., Feron, E., and Balakrishnan, V., Linear Matrix Inequalities in System and Control Theory. Philadelphia, PA: SIAM, 1994.

6. Cao, J., "Periodic oscillation and exponential stability of delayed CNNs," Physics Letters A, Vol. 270, pp. 157-163 (2000).

7. Gopalsamy, K. and He, X. Z., "Delay-independent stability in bidirectional associative memory networks," IEEE Transactions on Neural Networks, Vol. 5, pp. 998-1002 (1994).

8. Hale, J. K., Theory of Functional Differential Equations, Springer-Verlag, New York, 1977.

9. Has'minskii, R. Z., Stochastic Stability of Differential Equations, Sijthoff and Noordhoff, Alphen, 1981.

10. He, Y., Wu, M., She, J. H., and Liu, G. P., "Parameter-dependent Lyapunov functional for stability of time-delay systems with polytopictype uncertainties," IEEE Transactions on Automatic Control, Vol. 49, pp. 828-832 (2004)

11. Hu, J., Zhong, S., and Liang, L., "Exponential stability analysis of stochastic delayed cellular neural network," Chaos, Solitons \& Fractals, Vol.
27, pp. 1006-1010 (2006)

12. Liao, T. L. and Wang, F. C., "Global Stability for cellular neural networks with time delay," IEEE Transactions on Neural Networks, Vol. 11, pp. 1481-1484 (2000).

13. Liao, X. X. and Mao, X., "Exponential stability and instability of stochastic neural networks," Stochastic Analysis and Applicatios, Vol. 14, pp. 165-185 (1996).

14. Lu, C. Y., Su, T. J., and Tsai, J. S. H., "On robust stabilization of uncertain stochastic time-delay systems-an LMI-based approach," Journal of the Franklin Institute, Vol. 342, pp. 473-487 (2005).

15. Mao, X., Stochastic Differential Equations and Their Applications, Horwood, Chichester, 1997.

16. Verriest, E. I., "Behavior of stochastic neural networks with delays," Proceedings of the $37^{\text {th }}$ IEEE Conference on Decision and Control, Tampa, Florida USA, 1998.

17. Wan, L. and Sun, J., "Mean square exponential stability of stochastic delayed Hopfield neural networks," Physics Letters A, Vol. 343, pp. 306-318 (2005).

18. Wang, Z., Lauria, S., Fang, J., and Liu, X., "Exponential stability of uncertain stochastic neural networks with mixed time-delays," Chaos, Solitons and Fractals, Vol. 32, pp. 62-72 (2007).

19. Xu, S., Lam, J., Ho, D. W. C., and Zou, Y., "Novel global asymptotic stability criteria for delayed cellular neural networks," IEEE Transactions on Circuits and Systems-II: Express Briefs, Vol. 52, pp. 349-353 (2005).

20. Yue, D. and Han, Q. L., "Delay-dependent exponential stability of stochastic systems with time-varying delay, nonlinearity, and Markovian switching," IEEE Transactions on Automatic Control, Vol. 50, pp. 217 222 (2005).

21. Zhang, J., Shi, P., and Qiu, J., "Novel robust stability criteria for uncertain stochastic Hopfield neural networks with time-varying delays," Nonlinear Analysis: Real World Applications, Vol. 8, pp. 1349-1357 (2007).

22. Zhu, W. and $\mathrm{Hu}, \mathrm{J}$. ., "Stability analysis of stochastic delayed cellular neural networks by LMI approach," Chaos, Solitons \& Fractals, Vol. 29, pp. 171-174 (2006) 